\title{
Deficiency of immunity to poliovirus type 3 : a lurking danger?
}

\author{
Claudia Reinheimer*, Imke Friedrichs, Holger F Rabenau and Hans W Doerr
}

\begin{abstract}
Background: Europe was certified to be polio-free in 2002 by the WHO. However, wild polioviruses remain endemic in India, Pakistan, Afghanistan, and Nigeria, occasionally causing polio outbreaks, as in Tajikistan in 2010. Therefore, effective surveillance measures and vaccination campaigns remain important. To determine the poliovirus immune status of a German study population, we retrospectively evaluated the seroprevalence of neutralizing antibodies (NA) to the poliovirus types 1, 2 and 3 (PV1, 2, 3) in serum samples collected from 1,632 patients admitted the University Hospital of Frankfurt am Main, Germany, in 2001, 2005 and 2010.
\end{abstract}

Methods: Testing was done by using a standardized microneutralization assay.

Results: Level of immunity to PV1 ranged between 84.2\% (95\%Cl: 80.3-87.5), 90.4\% (88.3-92.3) and 87.5\% (85.4-88.8) in 2001, 2005 and 2010. For PV2, we found 90.8\% (87.5-90.6), 91.3\% (89.3-93.1) and 89.8\% (88.7-90.9), in the same period. Seroprevalence to PV3 was 76.6\% (72.2-80.6), 69.8\% (66.6-72.8) and 72.9\% (67.8-77.5) in 2001 and 2005 and 2010, respectively. In 2005 and 2010 significant lower levels of immunity to PV3 in comparison to PV1 and 2 were observed. Since 2001, immunity to PV3 is gradually, but not significantly decreasing.

Conclusion: Immunity to PV3 is insufficient in our cohort. Due to increasing globalization and worldwide tourism, the danger of polio-outbreaks is not averted - even not in developed countries, such as Germany. Therefore, vaccination remains necessary.

Keywords: Poliomyelitis, Vaccination, Seroepidemiology, Lack of immunity, Germany

\section{Background}

Poliovirus, the etiologic agent of paralytic poliomyelitis (hereinafter referred to as polio), is a positive-sensed single-stranded RNA virus, which belongs to the genus Enterovirus. Enteroviruses in turn are a species of small, pathogenic, icosahedral viruses belonging to the family Picornaviridae. Polioviruses are subdivided in three immunologically different serotypes: poliovirus type 1, 2 and 3 (hereinafter referred to as PV1, 2, 3). Since 1960, polio has been controlled by the use of live oral polio vaccine (OPV) or inactivated polio vaccine (IPV). The latter poses no risk of vaccine-associated paralytic polio and has been used in Germany since 1998 [1,2]. Polio usually affects children under the age of 5 years: paralytic polio has been seen in one of 200 cases, fatal cases are seen in about $5-10 \%$ of paralytic polio in developing countries [3]. The number of

\footnotetext{
* Correspondence: claudia.reinheimer@kgu.de

Institute for Medical Virology, University Hospital Frankfurt am Main, Paul Ehrlich-Straße 40, 60596 Frankfurt am Main, Germany
}

polio cases has been reduced by $>99 \%$, from an estimated number of 350,000 cases worldwide in 1988 [4] to 1,606 cases in 2009 [4] and 1,294 cases in 2010 [5]. While Germany was deemed to be polio-free by WHO on June, $21^{\text {st }}$ in 2002 [6], circulating wild polioviruses remain endemic in four major locations: Nigeria, Pakistan, Afghanistan and India $[4,7,8]$. The occurrence of polio outbreaks, such those reported in Tajikistan, Turkmenistan and Kazakhstan in 2010 [9,10] or Angola in 2007/2008 [11] underlines the necessity of vaccination campaigns for polio prevention. Epidemiological surveillance is also crucial to document polio-absence in polio-free countries. Between 1997 and 2010 Germany has taken part in the acute flaccid paralysis (AFP)-program, which was initiated to exclude a PV-infection by a two-time stool investigation in cases of AFP in children younger than 15 years. Because of low participation rates an alternative surveillance-program was initiated in Germany by the National Commission for Polio-Eradication in the Federal Republic of Germany
C Biomed Central 
(Nationale Kommission für die Polioeradikation in der Bundesrepublik Deutschland) and the Robert Koch-Institut (RKI), Berlin, Germany, in 2005. It is the so called Enterovirus-Surveillance, which bases on laboratory diagnostic clarification of viral meningitis or encephalitis by stool or cerebrospinal fluid (CSF). By Enterovirus-Surveillance Germany fulfils the obligation to prove to be and remain polio-free, as required by WHO. Up to date, no polio-caused AFP case has been detected in Germany [12]. The objective of our study was to describe the immunity status to PV1, 2, 3 of a German urban cohort 13 years after introduction of IPV and 9 years after elimination of polio in Germany. Therefore, we retrospectively evaluated the seroprevalence of neutralizing antibodies (NA) to PV1, 2,3 using microneutralization assay (MNA) in 1,632 routinely collected serum samples in the university-hospital of Frankfurt am Main, Germany. Furthermore, we quantitatively analyzed and evaluated the level of immunity to PV1, 2, 3 by titres on average.

\section{Methods}

In the years 2001, 2005 and 2010 we tested 411, 878 and 343 serum samples, respectively, for NA to PV1, 2, 3. The serum samples were obtained from patients admitted to the University Hospital of Frankfurt am Main, Germany (FRA).

As we conducted an unlinked anonymous retrospective study, patients' data regarding age and sex were available. However, data concerning nationality, residence or concomitant diseases were not available.

The samples were routinely screened for NA against PV1, 2, 3 by MNA. MNA and controls are performed by an in-house procedure according to WHO guidelines [13] using vaccine-PV-strains. Currently, trivalent IPVs are mainly produced using the poliovirus strains Mahoney (PV1), MEF-1 (PV2), and Saukett (PV3), grown in Vero cell line $[14,15]$ The strains were obtained from the German reference laboratory for poliomyelitis and enteroviruses at the Robert Koch Institut, Berlin. The MNA was performed as previously described [16]. Briefly: Sera were inactivated at $56^{\circ} \mathrm{C}$ for $30 \mathrm{~min}$ before use, diluted two-fold from $1: 10$ to $1: 1280$ and then incubated for $1 \mathrm{~h}$ at $37^{\circ} \mathrm{C}$ (in a $\mathrm{CO}_{2}$ incubator) with 100 tissue culture infective dose ${ }_{50}$ $\left(\mathrm{TCID}_{50}\right)$ of challenge virus (either PV1, 2 or 3 ). After the incubation period $50 \mu \mathrm{l}$ of the serum-virus suspension was added to $50 \mu \mathrm{l}$ of Vero cells suspension (African green monkey kidney, ATCC CCL-81). Cell controls and a reference serum of known PV1,2,3 neutralizing activity was included in each test to examine reproducibility of results. Each test serum was investigated in triplicate. After incubation for 5-7 days, the highest dilution of serum that prevents the development of virus induced cytopathogenic effects (CPE) was recorded. The NA titre corresponded to the reciprocal of this dilution. A serum sample was considered positive, if antibodies were present at a dilution $1: \geq$ 10 of the serum specimen.

Statistical analysis was done by using the $95 \%$ confidence interval $(95 \% \mathrm{CI})$ with a significance level of $p<$ 0.05 , Chi-Square test or the Fisher's exact test in the case of low sample numbers by using the program BIAS for Windows 8.3 (Epsilon Verlag, Hochheim Darmstadt 2007). Differences were regarded significant with an error probability of $p<0.05$. The Kruskal-Wallis test was used for analyzing the association of seroprevalence and age. Results are presented in modification of age groups defined by WHO. Modification in this context means consolidation of 2 or 3 age groups within a single because of low sample numbers in the age group recommended by WHO. We furthermore used three, previously defined [16] NA titre ranges and used them to quantify the level of immunity to PV1, 2, 3. Thus, low, medium and high immunity is given for titre ranges between 1:10-1:20 and 1:40-1:160 and 1:320-1:1280, respectively.

\section{Results}

A non-age-specific, overall evaluation of PV1, 2, 3-NA is shown in Figure 1. Seroprevalence (with $95 \%$ CI in brackets) of NA to PV1 amounts $84.2 \%$ (80.3-87.5), $90.4 \%(88.3-92.3)$ and $87.5 \%(85.4-88.8)$ in 2001,2005 and 2010, respectively. Highest levels of seropositivity were detectable for PV2 with 90.8\% (87.5-90.6), 91.3\% (89.3-93.1) and $89.8 \%(88.7-90.9)$ in 2001, 2005 and 2010, respectively. Lowest seroprevalences were observed for PV3: values ranged between $76.6 \%$ (72.2$80.6), 69.8 \%(66.6-72.8)$ and $72.9 \%(67.8-77.5)$ in 2001 and 2005 and 2010, respectively.

In Figure 2 the age-specific seroprevalence of NA to PV1, 2, 3 is illustrated. In the age group of 15-29 years highest level of seropositivity to PV1 (all values in [\%]: 90.2; 95\%CI: 82.7-95.2) and PV2 (93.1; 86.3-97.2) were recorded. Highest level of seroprevalence to PV3 (78.8; 66.9-87.8) was detected in the group aged $>30$ years. In comparison to PV1 and PV2, all the age groups show a lower level of seroprevalence of anti-PV3. In the group aged 15-29 years a significant lower seroprevalence to PV3 (72.5; 62.8-80.9) than to PV1 $(90.2 ; 82.7-95.2)$ and PV2 (93.1; 86.3-97.2) was detectable $(\mathrm{p}<0.05)$.

A qualitative analysis of the NA titres using three levels of immunity (low [1:10-1:20], medium [1:40-1:160], high [1: > 320]) was used to assess the level of immunity to PV1, 2, 3. No immunity is given for titres lower than 1:10. Figure 3 illustrates the distribution to these immunitylevels. It shows a decreasing number of PV3-"high immunity"-sera from 2001 to 2005 and 2010 while the percentage of samples with "no immunity" is significantly increased over years $(p=0.008$; see Figure 4$)$. 

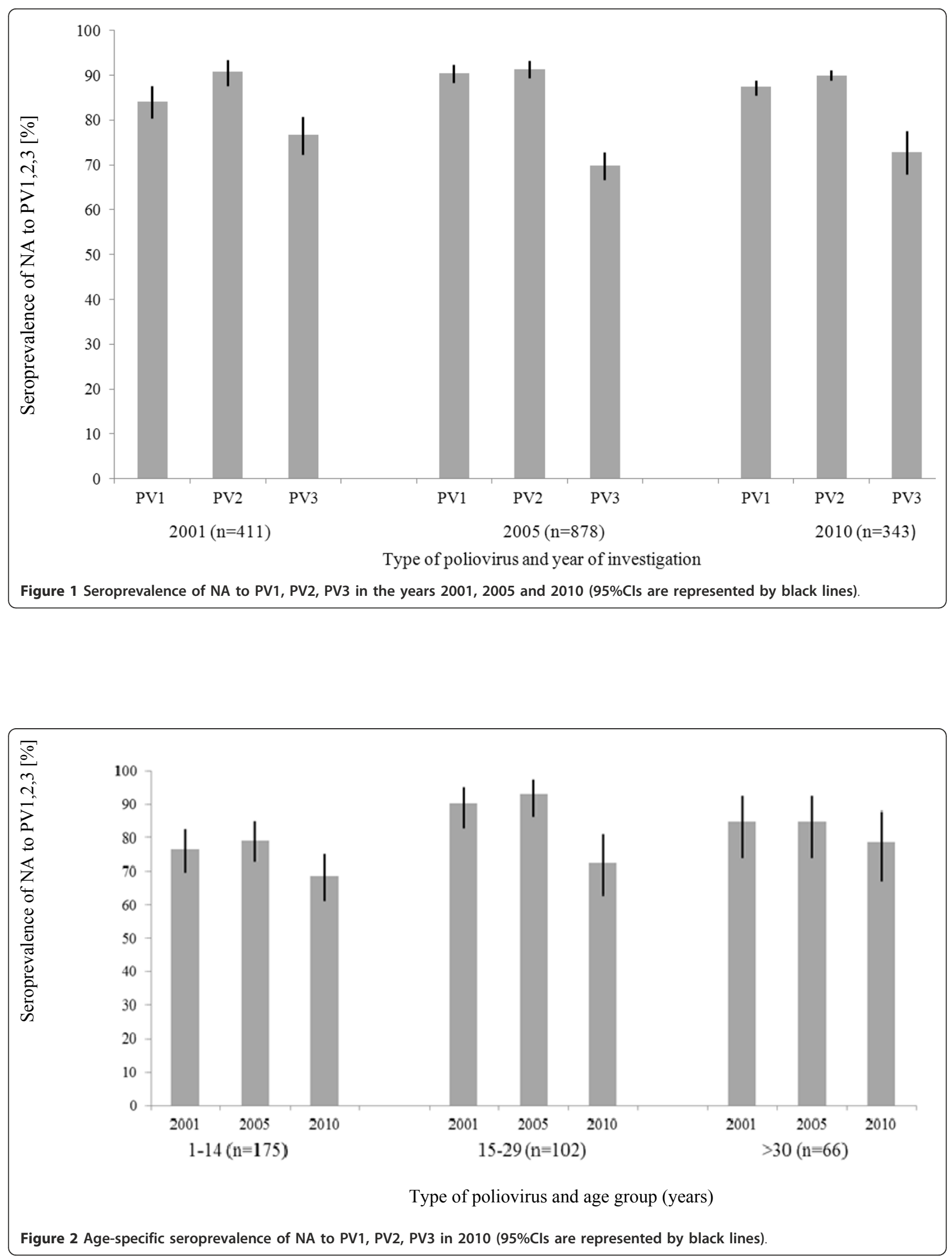

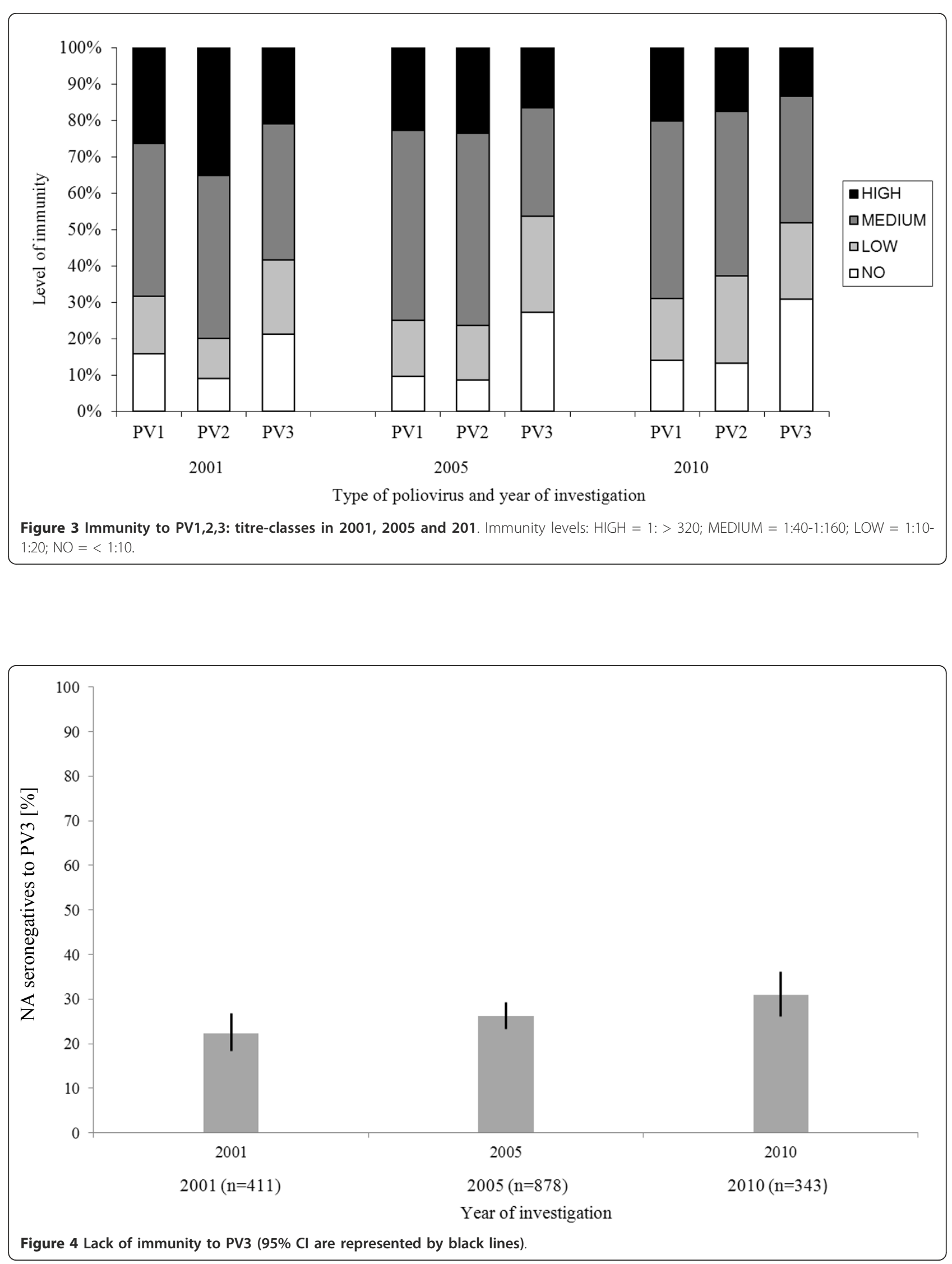


\section{Discussion}

From the epidemiological point of view, eradication of polio is a matter of urgency. The spread of wild polioviruses from endemic areas to polio-free countries remains a potential risk, as vaccination coverage rates can decrease, and vaccine-induced immunity can wane. For this reasons, vaccination campaigns and epidemiological surveillance are absolutely necessary to maintain and verify polio-absence in polio-free countries.

In our study population, we found a distinctive gap for PV3. This gap has previously been described by Weber et al. [16], Wicker et al. [17], Diedrich et al. in $[18,19]$, Franck et al. [20], but we found a further increase of this hiatus. In fact, also in our study the immunity level to PV3 is the lowest among all types of poliovirus [16-26]. This result can be explained by a reduced antigenicity immunogenicity of PV3 when compared to PV1 and PV2 [26]. This should be an aspect of interest in future vaccine development. An interesting aspect is that lowest seroprevalences of antibodies to PV1 - 3 were observed in the group aged 1-14 years (Figure 2). This phenomenon may be coherent with the implementation of IPV in Germany in 1998 [1,2]. When OPV was used, no such gap was observed [21].

Not only from this point of view but also in consideration of globalisation and worldwide tourism, polio remains an important theme: due to travel by air, importation of PV from endemic areas can occur within hours by air traffic. In Germany, 10-20\% of inhabitants are immigrants $[27,28]$, and may frequently invite or visit their relatives abroad. Consequently the risk of PVimport to Germany from endemic countries is present, and necessitates maintenance of an immune population until polio is eradicated globally.

Available data on immunological status to PV from other countries should be compared with caution, since determination of neutralizing antibodies is a biological assay with big deviations [28].

In Portugal 91.6\%, 94.2\% and 75\% of the tested persons had shown protective antibodies to PV 1,2,3, respectively, in 2002 [22]. Immigrants from less developed countries in North-East Italy were seropositive to PV 1, 2, 3 in $98.3 \%, 99.6 \%$ and $95.9 \%$, respectively [23]. Furthermore in Israel, 18 year-old soldiers recruited to the Israel Defence Forces tested for antibodies to PV1, 2, 3 were found to be protected in $98.7 \%, 99.6 \%$ and $96.4 \%$, respectively [24]. In Jilin Region, China, 95\%, 94.6\% and 92.3\%, had positive test results of NA against Polio type 1,2,3, respectively [25]. In comparison to our PV-seroprevalences (and also to former studies from Germany $[16,18-21])$ the results mentioned above are markedly higher. The reason for this phenomenon is unclear. In which way it is influenced by the way of titration, definition of the cut-off titre and counting of dilution (final dilution vs. serum dilution) remains unclear.

In a population of women attending antenatal in southern India, nearly $50 \%$ had shown no immunity (titres 1: $<8$ ) against polio, with the lowest levels for PV3 and 1 [29]. Nepal, a country three of four endemic countries, also shows hard effort in the fight against polio. However, while high acceptance of vaccinationprograms is documented [30], seroprevalence data are lacking. Seroprevalence surveys are necessary to identify population-categories at risk to be susceptible for polio, and to target specific vaccination activities to protect these groups. The availability of PV 1 and PV 3 monovalent vaccines vaccine could provide the global poliomyelitis eradication initiative with additional vaccine options [31], even though it could complicate decision making on vaccination policies.

Optimization of immunity to PV3 is a matter of urgency. As mentioned above and shown in Figure 1, 2, 3, 4, seroprevalence of NA to PV3 has been decreasing in Germany for several years. Therefore, continuing vaccination campaigns are as important as monitoring the population's immunity to sustain the present polio-free situation.

\section{Conclusions}

Despite intensive efforts in the fight against polio, it remains endemic in India, Pakistan, Afghanistan and Nigeria. Therefore, vaccination campaigns are as important as maintaining surveillance to monitor enteroviral events - including those countries, which are deemed to be polio-free by WHO. Even if the European countries are known to be polio-free the danger has not been completely averted: we observed a distinctive gap in immunity to PV3 in all age-groups and furthermore a decreasing immunity to PV, overall. Thus, we learn that vaccination against polio is absolutely necessary further on to preserve our present polio-free situation.

\section{Abbreviations \\ 95\%Cl: 95\% confidence interval; AFP: acute flaccid paralysis; CPE: cytopathogenic effect; CSF: cerebrospinal fluid; IPV: inactivated polio vaccine; MNA: microneutralization assay; NA: neutralizing antibodies; OPV: live oral polio vaccine; PV: Poliovirus; PV1: 2: 3: Poliovirus type 1: 2: 3; RKI: Robert Koch-Institut: Berlin: Germany; WHO: World Health Organisation.}

\section{Authors' contributions}

$C R$ coordinated data collection, wrote first draft and revisions of the paper, corresponding author; IF reviewed drafts of paper; HFR assisted with data collection, reviewed drafts of paper. HWD assisted with data analysis and reviewed drafts of paper; chief investigator of the study. All co-authors read and approved the final manuscript.

\section{Competing interests}

The authors declare that they have no competing interests.

Received: 31 May 2011 Accepted: 26 January 2012

Published: 26 January 2012 


\section{References}

1. Wattigney WA, Mootrey GT, Braun MM, Chen RT: Surveillance for poliovirus vaccine adverse events, 1991-1998: impact of a sequential vaccination schedule of inactivated poliovirus vaccine followed by oral poliovirus vaccine. Pediatrics 2001, 107(5):E83.

2. Windorfer A, Beyrer K: Poliomyelitis-why we must continue to vaccinate! MMW Fortschr Med 2005, 147(8), 36, 38, 40.

3. Roberts L: Infectious disease. Polio outbreak breaks the rules. Science 2010, 330(6012):1730-1731.

4. Centers for Disease Control and Prevention (CDC): Progress toward interruption of wild poliovirus transmission - worldwide, 2009. CDC MMWR Morb Mortal Wkly Rep 2010, 59(18):545-550.

5. , [http://www.polioeradication.org/dataandmonitoring/Poliothisweek.aspx] as of Wednesday 23 March 2011.

6. Robert Koch-Institut, Epidemiologisches Bulletin 26/2002.

7. Nathanson N, Kew OM: From emergence to eradication: the epidemiology of poliomyelitis deconstructed. Am J Epidemiol 2010, 172(11):1213-1229.

8. WHO Wkly Rec 2010 85: Progress toward poliomyelitis eradication India, January 2009-October 2010. CDC MMWR Morb Mortal Wkly Rep 2010, 59(48):1581-1585.

9. Centers for Disease Control and Prevention (CDC): Outbreaks following wild poliovirus importations - Europe, Africa, and Asia, January 2009September 2010. MMWR Morb Mortal Wkly Rep 2010, 59(43):1393-1399.

10. Macdonald N, Hébert PC: Polio outbreak in Tajikistan is cause for alarm. CMAJ 2010, 182(10):1013.

11. Kidd S, Goodson JL, Aramburu J, Morais A, Gaye A, Wannemuehler K, Buffington J, Gerber S, Wassilak S, Uzicanin A: Poliomyelitis outbreaks in Angola genetically linked to India: risk factors and implications for prevention of outbreaks due to wild poliovirus importationsa. Vaccine 2011, 29(21):3760-3766.

12. Reinheimer C, Rabenau HF, Berger A, Doerr HW: Diagnostic of Enteroviruses in clinical cases of aseptic meningitis in Frankfurt/Main 2006-2010. Klin Padiatr 2011, 223(4):221-226.

13. WHO: Manual for the virological investigation of polio Geneva: World Health Organization; 1997.

14. Tano Y, Shimizu H, Martin J, Nishimura Y, Simizu B, Miyamura T: Antigenic characterization of a formalin-inactivated poliovirus vaccine derived from live-attenuated Sabin strains. Vaccine 2007, 25(41):7041-7046.

15. Kew O, Sutter R, de Gourville E, Dowdle W, Pallansch M: Vaccine-derived polioviruses and the endgame strategy for global polio eradication. Annu Rev Microbiol 2005, 59:587-635.

16. Weber B, Rabenau H, Cinatl J, Maass G, Doerr HW: Quantitative detection of neutralizing antibodies against polioviruses and non-polio enteroviruses (NPEV) using an automated microneutralization assay: a seroepidemiologic survey. Zentralbl Bakteriol 1994, 280(4):540-549.

17. Wicker S, Rabenau H, Gottschalk R, Doerr HW, Allwinn R: Seroprevalence of vaccine-preventable and blood-transmissible viral infections (measles, mumps, rubella, VZV, polio, HBV, HCV, HIV) in medical students. Med Microbiol Immunol 2007, 196:145-150.

18. Diedrich S, Schreier E: Status of immunity against poliomyelitis. Polio serosurvey in 1993. Dtsch Med Wochenschr 1995, 120(8):239-244.

19. Diedrich S, Schreier E: The German Health Interview and Examination Survey for Children and Adolescents (KiGGS): state of immunity against poliomyelitis in German children. Bundesgesundheitsblatt Gesundheitsforschung Gesundheitsschutz 2007, 50(5-6):771-774.

20. Franck S, Allwinn R, Rabenau HF, Doerr HW: Epidemiological analysis of immunity to poliovirus after termination of an era of vaccination with OPV in Germany. An analysis of the German association against viral diseases (DVV). Zentralbl Bakteriol 1999, 289(4):475-481.

21. Maass $G$, Weber B, Doerr HW: The status of immunity against poliomyelitis. The 5th cooperative study of the german union to control viral diseases, Inc. Dtsch Med Wochenschr 1991, 116:1457-1462.

22. Pires de Miranda M, Carmo Gomes M, Rebelo de Andrade H: Seroprevalence of antibodies to poliovirus in individuals living in Portugal, 2002. Euro Surveill 2007, 12(6):E7-E8.

23. Trivello R, Ngatchu T, Marin V, Moretti G, Malatesta R, Maini P, Moschen ME, Baldo V, De Marzi L, Majori S, Puppo A, Renzulli G: Immunity status to polioviruses among non-European union immigrants in Veneto Region (North-East Italy). Ann Clin Lab Sci 1996, 26(2):154-159.
24. Grotto I, Handsher R, Gdalevich M, Mimouni D, Huerta M, Green MS, Mendelson E, Shpilberg O: Decline in immunity to polio among young adults. Vaccine 2001, 19(30):4162-4166.

25. Liu GY, Wang $L X$, Chang $X$ : Surveillance on poliomyelitis neutralization antibody level in the border areas in Jilin province. Zhongguo Yi Miao He Mian Yi 2009, 15(3):249-250.

26. Roivainen M, Montagnon B, Chalumeau H, Murray M, Wimmer E, Hovi T: Improved distribution of antigenic site specificity of poliovirusneutralizing antibodies induced by a protease-cleaved immunogen in mice. J Virol 1990, 64(2):559-562.

27. www.destatis.de/[...]/MigrationIntegration/Migrationshintergrund/Tabellen/ Content100/MigrationshintergrundLaender,templateld=renderPrint.psml as of Friday 01 April 2011.

28. Diedrich $\mathrm{S}$, Claus H, Schreier E: Immunity status against poliomyelitis in Germany: determination of cut-off values in International Units. BMC Infect Dis 2002, 2:2.

29. John J, Abraham AM, Muliyil J, John TJ, Deshpande JM, Kang G: Gap in the prevalence of neutralising antibodies to polioviruses in antenatal women in southern India. Trans R Soc Trop Med Hyg 2011, 105(3):179-180.

30. Shah $\mathrm{H}$ : A cluster survey for determination of regular vaccination coverage among children. JNMA J Nepal Med Assoc 2008, 47(170):91-93.

31. Grassly NC, Wenger J, Durrani S, et al: Protective efficacy of a monovalent oral type 1 poliovirus vaccine. Lancet 2007, 369(9570):1356-1362.

\section{Pre-publication history}

The pre-publication history for this paper can be accessed here: http://www.biomedcentral.com/1471-2334/12/24/prepub

doi:10.1186/1471-2334-12-24

Cite this article as: Reinheimer et al: Deficiency of immunity to poliovirus type 3: a lurking danger? BMC Infectious Diseases 2012 12:24.

\section{Submit your next manuscript to BioMed Central and take full advantage of:}

- Convenient online submission

- Thorough peer review

- No space constraints or color figure charges

- Immediate publication on acceptance

- Inclusion in PubMed, CAS, Scopus and Google Scholar

- Research which is freely available for redistribution

Submit your manuscript at www.biomedcentral.com/submit
Biomed Central 